\title{
Determination of metallic complexing capacities of the dissolved organic material in seawater*
}

\author{
LUIS M. LAGLERA-BAQUER, MELCHOR GONZÁLEZ-DÁVILA \\ and J. MAGDALENA SANTANA-CASIANO \\ Departmento de Química, Universidad de Las Palmas de Gran Canaria. 35017 Las Palmas de Gran Canaria, Spain.
}

\begin{abstract}
SUMMARY: The use of the Langmuir isotherm for the study of the complexing properties of functional groups present both in dissolved organic material and on biological surfaces in aquatic systems needs a heterogeneous model and an iterative linear regression solution. The method proposed previously by van den Berg is improved by replacing the expression used to obtain the complexing capacity and the conditional stability constant of the functional groups with stronger trace metal affinity, for the linear expression of the Langmuir isotherm proposed by Scatchard. The result is also an iterative linear regression process, which gives a convergent solution, together with better statistical weight distribution of the initial metallic titration data. It also fits better with the titration data obtained at very low dissolved metallic ion concentrations.
\end{abstract}

Keywords: Langmuir isotherm, complexation, trace metals, complexing capacities.

\section{INTRODUCTION}

The reactivity and bioavalibility of trace metals in aquatic systems are regulated by their speciation (Sunda and Guillard, 1976; Brand et al., 1983). In fact, the results obtained through bioassay experiments (Sunda and Guillard, 1976; Anderson and Morel, 1978, 1982; Sunda and Ferguson, 1983; Folsom et al., 1986) indicate that the chemical parameters controlling metal-biosurface interactions are free metal ion activities. The dissolved organic material has an important biological role to play in avoiding this kind of interaction. According to previous studies for several metals $(\mathrm{Cu}, \mathrm{Pb}, \mathrm{Fe}, \mathrm{Zn}$, etc) the metal-dissolved organic material (DOM) com-

\footnotetext{
*Received October 1, 1999. Accepted July 17, 2000.
}

plexes are predominant in natural aquatic systems (Coale and Bruland, 1988, 1990; Capodaglio et al., 1990; Wu and Luther, 1995; Rue and Bruland, 1995; Xue and Sigg, 1993). At present, it is believed that this complexing material proceeds essentially from the release of extracellular organic matter by marine phytoplankton (Imber et al., 1985; Zhou and Wangersky, 1985, 1989; Seritti et al., 1986; Moffett et al., 1990; Moffett 1995). The functional groups with affinity to bind trace metals located on the surface of the organisms play a similar role in metal speciation depending on the medium biomass.

Significant efforts have been made to describe the stability of the metal-binding site reaction of algal exudate (Zhou and Wangersky, 1985, 1989; Gerringa et al., 1995), functional groups from biological surfaces (Xue and Sigg, 1990; Xue et al., 


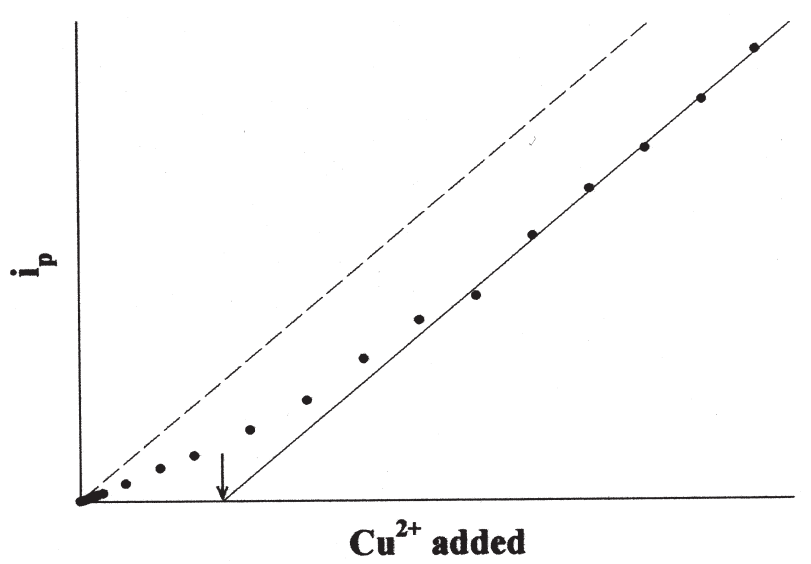

FIG. 1. - Example of a copper titration data set obtained from a natural aquatic medium. The dashed line is the result obtained in the absence of complexing functional groups. The continuous line corresponds to the estimation of the sensitivity of the analytical technique. The arrow shows how to estimate the complexing capacity of the sample directly from the graph.

1988; Santana et al., 1995) and the organic material pool of seawater (van den Berg, 1984; van den Berg and Donat, 1992; Coale and Bruland, 1988, 1990; Capodaglio et al., 1990) plus fresh water systems (Xue and Sigg, 1993). In spite of the large number of works that have been published, the origin and nature of the organic dissolved material with affinity for trace metals in aquatic natural media, the complexing characteristics of plankton exudates, and the importance of the microorganism in the metal speciation regulation are still largely unknown.

The most usual method for the evaluation of the complexing capacity of a sample and the stability constant for a specific metal of the functional groups, which take part in the binding reaction is to titrate the sample with the metal. A selective and sensitive analytical technique must be used to monitor the metal speciation during the titration process. As the metal concentration in the sample increases, the functional groups are complexed with the metal ion until they are saturated. After this point, the metal concentration, which is not organically complexed increases linearly (Fig. 1). Some data treatments have been suggested in order to come to a description of the metal speciation in natural media through the determination of the binding characteristics of the complexing sites found in the system. In the present work, we propose a new method for improving the definition of these binding characteristics. We will present examples obtained with diatom culture medium after filtering and with cellular suspensions via the use of voltammetric techniques.

\section{THEORY}

\section{Solution of voltammetric titration procedures}

The solution used to describe the complexing characteristics of an aquatic medium presumes a single 1:1 binding mechanism.

$$
\mathrm{L}_{\mathrm{x}}-+\mathrm{M}^{\mathrm{n}+} \Leftrightarrow \mathrm{L}_{\mathrm{x}}-\mathrm{M}
$$

where $\mathrm{L}_{\mathrm{x}}$ designates a binding site (referring to a biological surface or to an organic dissolved molecule) and $\mathrm{M}^{\mathrm{n}+}$ represents a free dissolved metal cation. A proton or a major cation of the medium $\left(\mathrm{Na}^{+}, \mathrm{Mg}^{2+}, \mathrm{Ca}^{2+}\right.$, etc. for seawater) may also be initially bonded to the binding site.

The two parameters which describe the complexing reaction are the total complexing capacity (concentration of the total functional groups present both in solution and on the biological surface, with affinity for the metal cation used in the titration) and the conditional stability constant, defined respectively as:

$$
\begin{gathered}
{\left[L_{X}\right]_{T O T}=\left[\mathrm{L}_{\mathrm{x}}^{\prime}\right]+\left[\mathrm{ML}_{\mathrm{x}}\right]} \\
K^{\prime}{ }_{M L_{X}}=\left[\mathrm{ML}_{\mathrm{x}}\right] /\left(\left[\mathrm{M}^{\mathrm{n}+}\right]\left[\mathrm{L}_{\mathrm{x}}^{\prime}\right]\right)
\end{gathered}
$$

where $\mathrm{L}_{\mathrm{x}}$ is the concentration of natural binding sites which are not complexed by the metal. The value of $\mathrm{K}^{\prime}$ depends upon the solution composition $(\mathrm{pH}$, major cation concentrations) and is therefore conditional for the medium. The latter two formulae give the final expression known as the Langmuir isotherm. This represents a saturation curve.

$$
\left[M L_{X}\right]=\frac{K^{\prime} \cdot\left[L_{X}\right]_{T O T} \cdot\left[M^{n+}\right]}{1+K^{\prime} \cdot\left[M^{n+}\right]}
$$

where the left side of the equation gives the concentration of metal bonded by the dissolved organic material in complexation experiments with natural aquatic samples. In adsorption experiments with biological surfaces of plankton cells, this left side of the equation represents the cellular surface coverage, which is the percentage for a single cell of the binding sites bonded with metal ions.

With a non-linear fit of this equation, it is possible to obtain the complexing characteristics of the medium (Gerringa et al., 1995). However, the most usual way to come to a precise definition is to build linearizing plots. Scatchard (1949) proposed the following expression (which is plotted in Fig. 2a accordingly): 

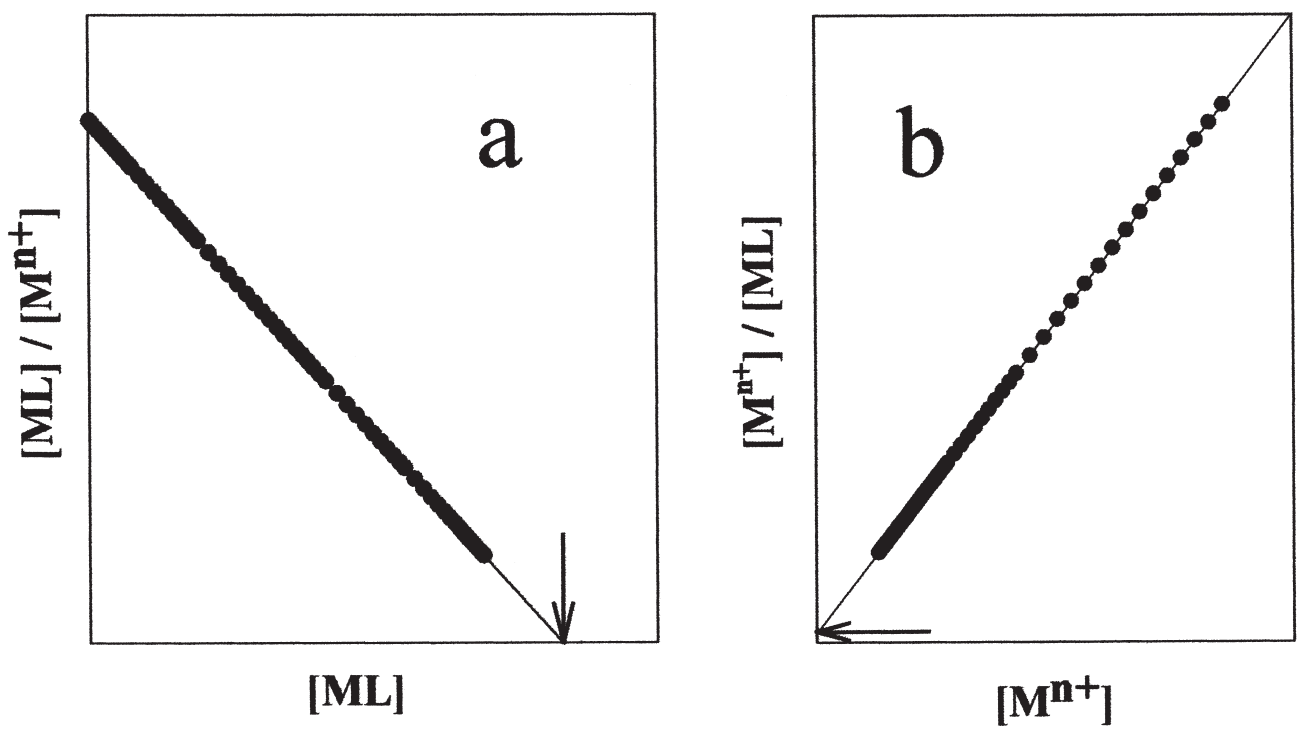

FIG. 2. - (a) Scatchard linearising plot for a model data set. The arrow shows the ligand concentration of the theoretical medium. (b) Van den Berg-Ružić-Lee plot of the same data set.

$$
\left[M L_{X}\right] /\left[M^{n+}\right]=K^{\prime}\left(\left[M L_{X}\right]+\left[L_{X}\right]\right)
$$

The conditional stability constant is calculated from the slope of the plot, and the total ligand concentration from the intersection with the y-axis.

Van den Berg (1982), Ružić (1982) and Lee (1983) proposed another linearising plot following the expression:

$$
\frac{\left[M^{n+}\right]}{\left[M L_{X}\right]}=\frac{\left[M^{n+}\right]}{\left[L_{X}\right]}+\frac{1}{K_{M L_{X}} \cdot\left[L_{X}\right]}
$$

which is the result after plotting the left side of the last expression versus $\left[\mathrm{M}^{\mathrm{n}+}\right]$, as shown in Figure $2 \mathrm{~b}$. The total concentration of binding sites is obtained from the slope, and the conditional stability constant from the intersection with the y-axis.

Both linearising plots yield the same solution for the complexing capacity and conditional stability constant, working with ideal titration data because they are algebraically equivalent. However, the error scatters associated with the analytical techniques has different influences on the cited plots. In the Scatchard plot, the greater the statistical weight of the data plotted, the lower the total metal concentration. The van den Berg-Ružić gives the greatest statistical weight to the last points of the titration. This yields different results when we use titration data from real solution experiments. Several authors have compared the accuracy of the two linearising plots (Apte et al., 1988; Ružić 1982, 1996; Miller and Bru- land, 1997) and have concluded that the van den Berg-Ružić plot is more suitable for determining complexing capacities. This is because it is more sensitive in the region where the points are subject to the smallest relative errors, and only in this plot it is possible to discern whether the appearance of nonlinearities is due to the heterogeneity of the binding sites or to kinetic effects. If the data causes poor fitting of the van den Berg-Ružić method, this usually indicates a problem with the data and suggests that incorrect results may be obtained. Ružić (1996) indicates the usefulness of the Scathard plot for studying the heterogeneity of the binding sites of the medium because they appear in the region of lower metal concentrations, where this plot is more sensitive.

When a natural aquatic medium (freshwater or seawater) is analysed using a high sensitivity method, both linearising methods yield curve plots due to the great heterogeneity of the binding sites of the biological surfaces and dissolved organic materials. This is the case for a vast variety of trace metals such as $\mathrm{Cu}$ (van den Berg, 1984, van den Berg and Donat, 1992; Coale and Bruland, 1988, 1990), $\mathrm{Pb}$ (Capodaglio et al., 1990) and $\mathrm{Fe}$ (Wu and Luther, 1995; Rue and Bruland, 1995). It is necessary to consider more than one kind of complexing site. Usually a two-site model, which considers the existence of one class of high affinity ligand for the metal studied $\left(\mathrm{L}_{1}\right)$ and one class of ligands defined by a low conditional stability constant $\left(\mathrm{L}_{2}\right)$, fits well to the titration data. The Langmuir isotherm for two kinds of binding sites takes the form: 


$$
[M L]=\frac{\left[L_{1}\right] \cdot K_{1} \cdot\left[M^{n+}\right]}{1+K_{1}\left[M^{n+}\right]}+\frac{\left[L_{2}\right] \cdot K_{2} \cdot\left[M^{n+}\right]}{1+K_{2}\left[M^{n+}\right]}
$$

Direct graphical solutions for the value of the four complexing parameters have been suggested (Sposito, 1982), but this process means that the solution given for every kind of ligand is not independent of them. This is due to the two premises worked upon: first, the high affinity ligand class is responsible for the metal binding at total low metal concentrations; and second, in the last part of the titration curve, the kind of ligand with low affinity for the metal ion is the only binding species involved in the complexing reaction. However, at any metal concentration, both classes of ligand will bind a fraction, and the solutions for the four complexing parameters are affected by the concentration of metal complexed by the ligand class not considered.

Van den Berg (1984) proposed an iterative method to resolve this problem. In this method, approximated values of $\left[\mathrm{L}_{1}\right]$ and $\mathrm{K}_{\mathrm{L} 1}$ are estimated via the use of the van den Berg-Ružić linearising treatment with the initial titration data (in which the metal is preferentially bound by the high affinity ligand). Via the use of the mass balance for the ligand, and the relationship between the concentrations of free metal cation and metal bound with the high affinity ligand given by the Langmuir isotherm defined for a single ligand class, it is possible to estimate the metal concentration bound by the low affinity ligand. The next step is to generate a new van den Berg-Ružić plot with the last points of the titration. At these points, most of the metal is bound by the low affinity metal, once the concentration bound by the high affinity ligand has been subtracted. Then it is possible to obtain estimations of $\left[\mathrm{L}_{2}\right]$ and $\mathrm{K}_{\mathrm{L} 2}$ and to use them to get new approximated values for the complexing parameters of the high affinity ligand class. The process continues until convergent solutions are reached.

The solutions of the complexing parameters given by the iterative method for one ligand class are independent from the values obtained for the other binding site class. This method has the disadvantage that the solutions reached for the complexing capacity and conditional stability constant of one ligand class are not independent of each other and overestimates of one produce the opposite effect in the other. Another problem found is that the results obtained are dependent on the number of data points selected to estimate every kind of ligand.

\section{RESULTS AND DISCUSSION}

The van den Berg iterative method fits well with the sets of model and real titration data. However, some problems are associated with this iterative method. The initial points obtained using the van den Berg-Ružić linearising treatment depend on the data scatter due to analytical errors, and may yield a linear regression with a negative y-axis intercept (see the proximity of the linear regression intercept with the y-axis, marked with the arrow, with respect to the graph origin in Fig. 2b). This pre-supposes the appearance of negative values for the stability constant and the impossibility of reaching a convergent solution (see Table 1). This problem does not appear using the Scatchard linearizing plot.

Furthermore, the use of the van den Berg-Ružić linearising method leads to a higher statistical weight when the metal is more bonded by the low affinity ligand. It works on the basis that the initial data, without any metallic addition to the sample and the maximum relative importance of the $M-L_{1}$ species in the metal speciation, has the minimum statistical weight in the estimation process of the complexing parameters of the high stability constant ligands. The concentration of $\mathrm{M}-\mathrm{L}_{1}$ is calculated by subtracting the analysed labile metal concentration and the $M-L_{2}$ concentration from the total metal concentration. The $\mathrm{M}-\mathrm{L}_{2}$ concentration is obtained via the estimation, which yields a linear regression of the last points of the van den Berg-Ružić plot.

$$
\left[M L_{1}\right]=[M]_{T O T}-\left[M L_{2}\right]-i_{p} / S
$$

For the titration data set, the lower the percentage of the $\mathrm{M}-\mathrm{L}_{1}$ species with respect to the total metal concentration (which occurs as the titration procedures advances), the greater the possible error introduced in the estimating iterative process through the analysis of the labile fraction and the estimation of the complexing parameters of the low affinity ligand class. This is due to the fact that a small value $\left(\left[\mathrm{ML}_{1}\right]\right)$ is the result of subtracting two greater numbers subjected to errors from the total metal concentration. If the accuracy of the analytical method is sufficient (which means that there must be a fair estimation of the free metal concentration before addition), the most appropriate points for determining the complexing characteristics of the high affinity ligands present in the analysed medium are those that are as close as possible to the beginning of the titration procedure. 
TABLE 1. - Complexing parameter values for three differents copper data titrations of seawater samples from open ocean and from culture mediums of $P$. tricornutum. A two-binding site model was adopted. Two iterative methods were used: the method proposed by Van den Berg (1984) and the mixed method suggested in the present work. Different values come from changes in the number of data used to obtain the complexing characteristics of the high stability ligands.

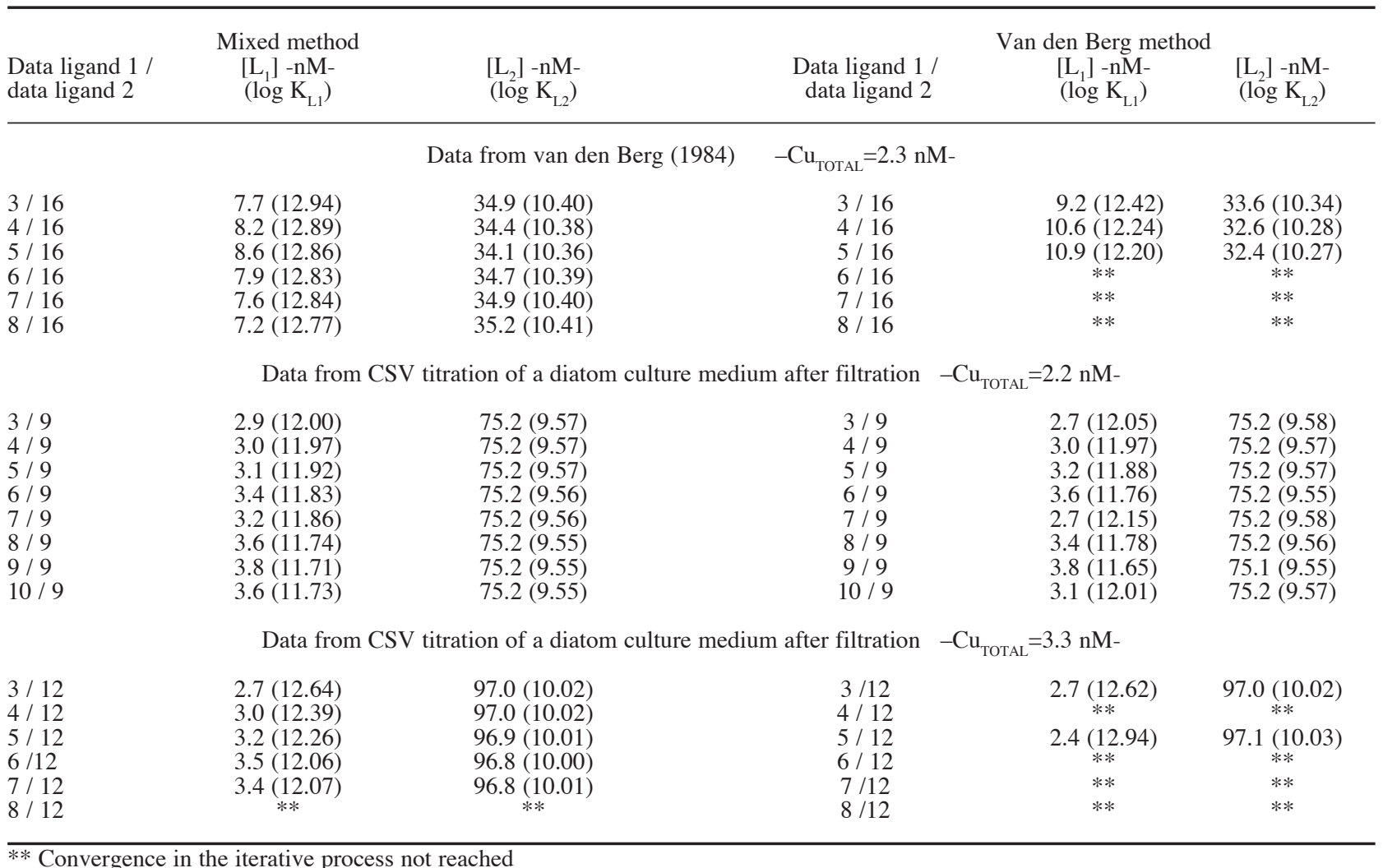

For this reason, in order to avoid possible interruptions in the iterative technique cited, a mixed iterative linearising method is proposed. The Scatchard plot is used in the initial points of a titration data set to estimate the complexing characteristics of the high affinity ligands. The van den BergRužić-Lee plot is necessary to obtain a fair estimation of the complexing properties of the low affinity binding sites of the medium. This method requires accuracy in the estimation of the initial data values because it gives the greatest statistical weight to points subject to significant relative errors in the analytical stage.

To visualise how this method can improve the fit of the initial values, the data titration set obtained by van den Berg (1984) is represented by the linearising plots shown earlier in Figures 3 and 4. In the graphs, the result of building a process using only the Scatchard linearising method is also included to show how a mixed method reduces the disadvantages associated with the other linearising methods. In all the curves shown in Figures 3 and 4, the five initial data were taken from the data set to obtain the

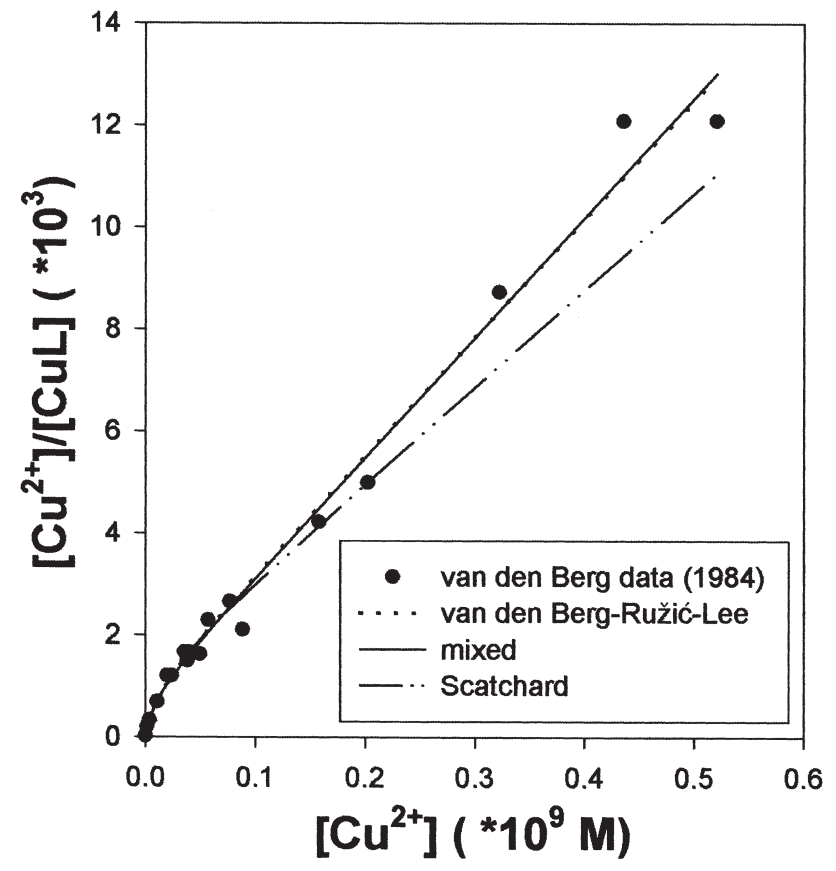

Fig. 3. - Van den Berg-Ružić linearising plot of the data set from van den Berg (1984). The lines correspond to three different iterative methods for resolving the complexing parameters for a two binding-site model. 


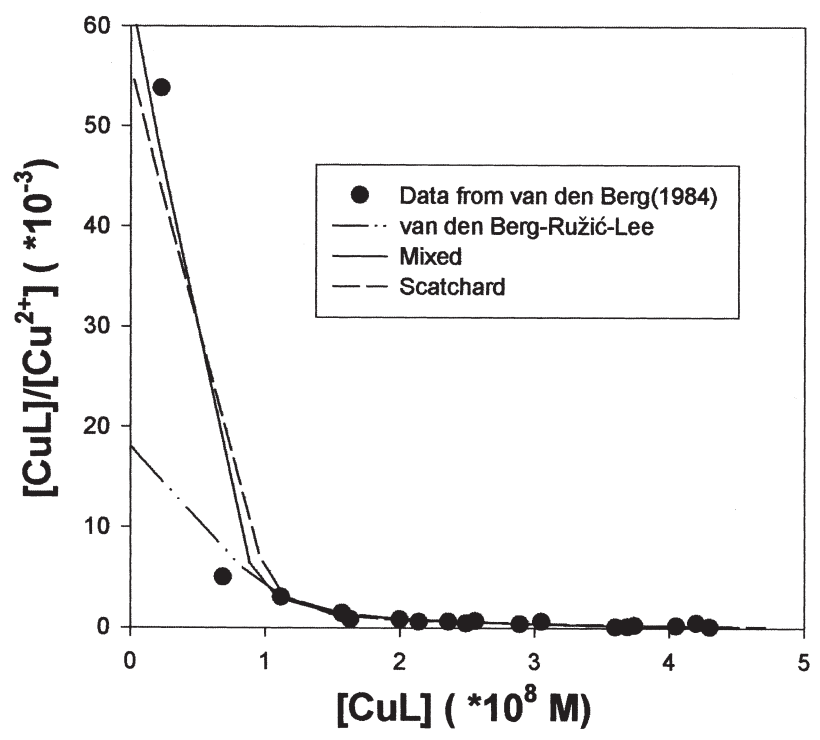

FIG. 4. - Scatchard linearising plot of the data set from van den Berg (1984). The lines correspond to three different iterative methods for resolving the complexing parameters for a two binding-site model. complexing characteristics of the high affinity ligand. In the same way, the last sixteen pieces of data were taken to reach a solution for the complexing parameters of the low affinity ligand class in all the iterative processes. This means that the only differences which appear in the results are due to the different distribution given to the statistical weight of the titration data during the iterative routine.

Table 2 shows the values obtained following the three methods for the complexing capacities and conditional stability constants. The van den BergRužić plot is optimal for showing the fit of the method to the last points of the titration where the metal bonded by the weak ligand is the major component of the organic complexed fraction. The methods using the van den Berg-Ružić-Lee linearising treatment for the estimation of the cited ligand fit this part of the curve well.

To study the fit of the initial titration data it is necessary to observe the Scatchard plot (see Fig. 4),

TABLE 2. - Complexing parameter values obtained for the titration data set of van den Berg (1984). Results obtained taking 5 piece of data for the estimation of $\mathrm{L} 1$ parameters and 16 pieces of data to calculate the L2 binding parameters $\left(\mathrm{a}^{\prime}=304.5\right)$.

\begin{tabular}{lcccc}
\hline Linearising method used for the iterative process & {$\left[\mathrm{L}_{1}\right] * 10^{9} \mathrm{M}$} & $\log \mathrm{K}_{\mathrm{L} 1}$ & {$\left[\mathrm{~L}_{2}\right]^{*} 10^{8} \mathrm{M}$} & $\log \mathrm{K}_{\mathrm{L} 2}$ \\
\hline Van den Berg-Ružić-Lee & $10.9 \pm 0.4$ & $12.20 \pm 0.16$ & $3.24 \pm 0.17$ & $10.27 \pm 0.1$ \\
Scatchard & $10.1 \pm 0.5$ & $12.74 \pm 0.11$ & $4.29 \pm 0.5$ & $10.08 \pm 0.09$ \\
Mixed & $8.6 \pm 0.3$ & $12.86 \pm 0.1$ & $3.41 \pm 0.16$ & $10.36 \pm 0.1$ \\
\hline
\end{tabular}
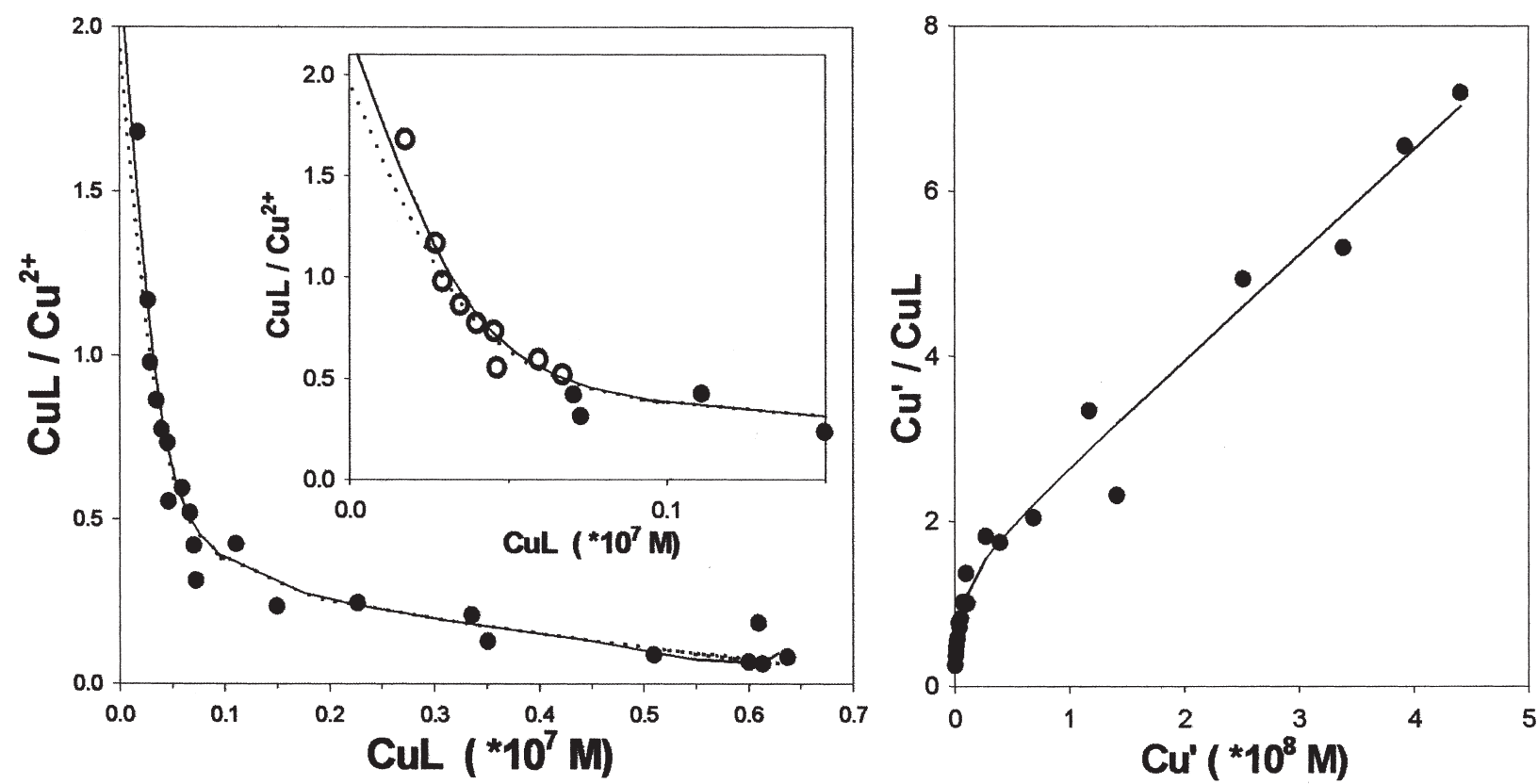

FIG. 5. - Scathard and van den Berg-Ružić linearising plot of the second data set shown in Table 1 if we take 9 pieces of data for the estimation of both ligand classes. The Scatchard plot includes an enlarged view of the 13 initial data to compare the accuracy of the fit resulting from the van den Berg and mixed iterative methods. - - - mixed iterative method ......... van den Berg iterative model. The dotted circles in the left graph are the selected values in both iterative processes. 


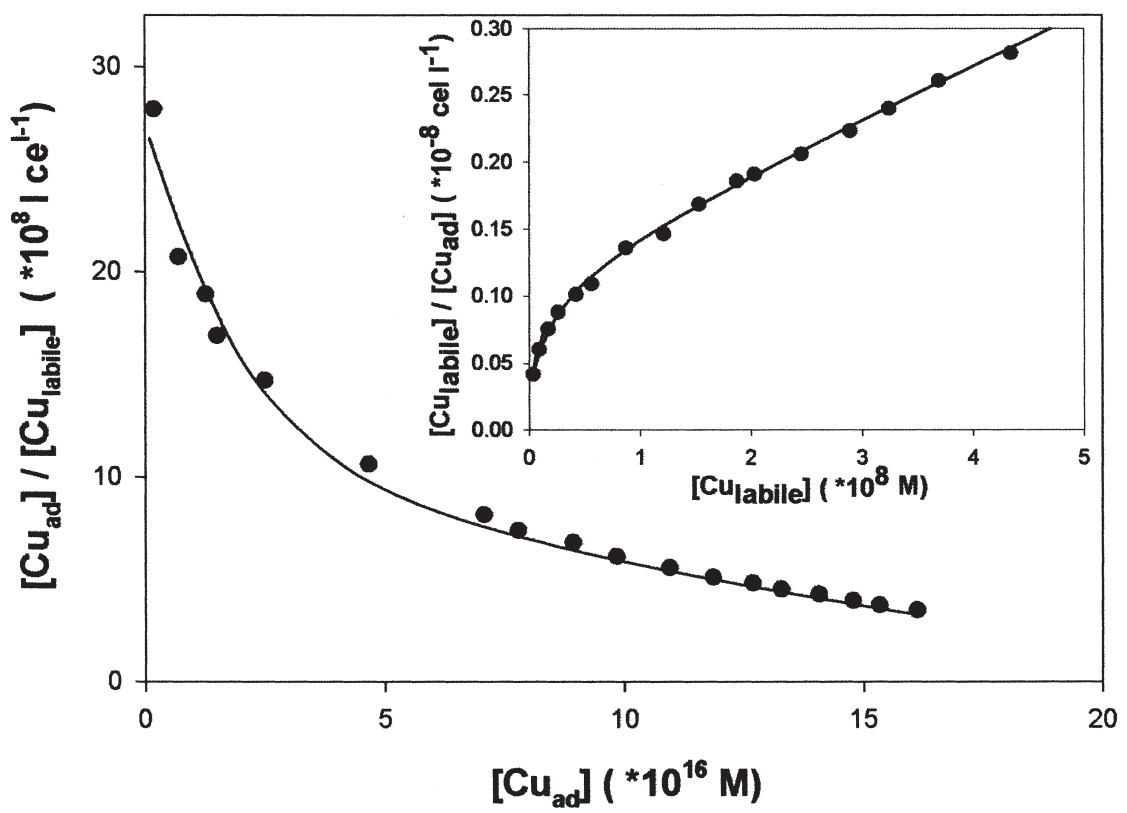

FIG. 6. - Application of the mixed iterative model to the estimation of the complexing characteristics for the surface groups of the diatom $P$. tricornutum assuming a two kinds of binding sites. The graphs correspond to the Scatchard linearising plot and van den Berg-Ružić-Lee plot (enclosed) of the same data set.

where we can see that only the iterative methods using the Scatchard linearising treatment for the estimation of the complexing parameters of the strong binding ligand fit well to the initial value.

Table 1 shows the results obtained for three titration data sets operating with the iterative method as proposed by van den Berg (1984) and with the mixed iterative routine explained before. The Table shows how the number of titration data selected for the estimation of a ligand class affects the result. The first method cited applied using the data provided by van den Berg (1984) presents convergence problems due to negative $\mathrm{y}$-axis intercepts when more than five of the titration data are taken. The mixed method proposed presents no problems of convergence with the data sets selected. The values obtained for the complexing characteristics of the high affinity ligand type are less dependent on the number of titration data taken for the estimation (as the number of data selected is increased, the greater statistical weight remains constant with respect to the few initial data and the estimation process suffers fewer changes).

Figure 5 shows the results obtained through both iterative methods for the second data set presented in Table 1 if we take 9 pieces of data for the estimation of both ligand classes. This graph also shows a better fit in the initial values if the mixed model is utilised.

Table 1 shows a progressive decrease in the conditional stability constant of the high affinity ligand class as the number of data taken is great. This is due to the heterogeneity of the organic material of natural environments. Within the same ligand class, the initial metal additions lead to the saturation of the binding sites with greatest affinity for the metal, the places with lower complexing tendency remaining unbound. This decreasing effect varies according to the relationship between the total metal concentration in the original medium and the concentration of the strong ligands present. This means that if we wish to obtain a trustworthy description with iterative linearising techniques of the binding characteristics of strong complexing sites, their concentration must be higher than the total metal concentration.

Figure 6 shows the accuracy reached with the mixed iterative method to fit data sets coming from experiments of metal adsorption onto biological surfaces of Phaeodactylum tricornutum (GonzalezDavila et al., 2000). The good agreement, and the more reliable fitting, suggest that the proposed method may indeed be a reasonable adaptation of the fitting methods.

\section{CONCLUSIONS}

The linearising iterative method proposed by van den Berg to fit titration data set from metal heterogeneous complexing media seems to yield a good description of the binding characteristics of the 
weak binding functional groups present in aquatic samples. However, this behaviour in the estimation of the complexing parameters of the strong binding ligands can be improved by replacing the use of the van den Berg-Ružić linearising treatment with the Scatchard linearising method for the initial titration data. The result is a method which is algebraically equivalent, involves fewer problems to reach a convergent solution, is less dependent on the number of data selected for the estimation of $\left[\mathrm{L}_{1}\right]$ and $\mathrm{K}_{\mathrm{L}}$, and gives greater statistical weight to those data in which the metal is bonded to a larger extent by highest affinity ligands when their binding characteristics are calculated. A correct estimation of the conditional stability constant of the high affinity ligands is needed because the latter are responsible for over 90\% of the metal speciation for several trace metals in aquatic systems $(\mathrm{Cu}, \mathrm{Fe})$. Without accuracy in this estimation, it is not possible to obtain reliable concentration data for the reactive species of the free metal ion.

\section{REFERENCES}

Anderson, D.M. and F.M.M. Morel. - 1978. Copper sensitivity of Gonyaulax tamarensis. Limnol. Oceanogr., 23: 283-295.

Anderson, D.M. and F.M.M. Morel. - 1982. The influence of aqueous iron chemistry on the uptake of iron by the coastal diatom Thalassiosira weissflogii. Limnol. Oceanogr., 27: 789-813.

Apte, S.C., M.J. Gardner and J.E. Ravenscroft. - 1988. An evaluation of voltammetric titration procedures for the determination of trace metal complexation in natural waters by use of computer simulation. Anal. Chim. Acta, 212: 1-21.

Brand, L.E., W.G. Sunda and R.R.L. Guillard. - 1983. Limitation of marine phytoplankton reproductive rates by zinc, manganese and iron. Limnol. Oceanogr., 28: 1182-1198.

Capodaglio, G., K.H. Coale and K.W. Bruland. - 1990. Lead speciation in surface waters of the Eastern North Pacific. Mar. Chem., 29: 221-233.

Coale, K.H. and K.W. Bruland. - 1988. Copper complexation in the Northeast Pacific. Limnol. Oceanogr., 33(5): 1084-1101.

Coale, K.H. and K.W. Bruland. - 1990. Spatial and temporal variability in copper complexation in the North Pacific. Deep-Sea Res., 37(2): 317-336.

Folsom, B.R., N.A. Popescu and J.M. Wang. - 1986. Comparative study of aluminum and copper transport and toxicity in an acid-tolerant freshwater green alga. Environ. Sci. Technol., 20: 616-620.

Gerringa, L.J.A., P.M.J. Herman and T.C.W. Poortvliet. - 1995. Comparison of the linear van den Berg / Ružić transformation and a non-linear fit of the Langmuir isotherm applied to $\mathrm{Cu}$ speciation data in the estuarine environment. Mar. Chem., 48: 131-142.

Gerringa, L.J.A., J.W. Rijstenbil, T.C.W. Poortvliet, J. van Drie and M.C. Schot. - 1995. Speciation of copper and responses of the marine diatom Dytilum brightwellii upon increasing copper concentrations. Aquatic Toxicol., 31: 77-90.

González-Dávila, M., J.M. Santana-Casiano and L.M. Laglera. - 2000. Copper adsorption in diatom cultures. Mar. Chem., in press.

Imber, B.E., M.G. Robinson, A.M. Ortega and J.D. Burton. - 1985. Complexation of zinc by exudates from Skeletonema costatum grown in culture. Mar. Chem., 16: 131-139.

Miller L.A. and K.W. Bruland. - 1997. Competitive equilibration techniques for determinig transition metal speciation in natural waters: Evaluation using model data. Anal. Chim. Acta, 343: 161-181.

Moffet J.W. - 1995. Temporal and spatial variability of copper complexation by strong chelators in the Sargasso Sea. DeepSea Res., 42(8): 1273-1295.

Moffet J.W., R.G. Zika and L.E. Brand. - 1990. Distribution and potencial sources and sinks of copper chelators in the Sargasso Sea. Deep-Sea Res., 37(1): 27-36.

Rue,E.L. and K.W. Bruland. - 1995. Complexation of iron (III) by natural organic ligands in the central North Pacific as determined by a new competitive ligand equilibration / adsorptive cathodic stripping voltammetric method. Mar. Chem., 50: 117138.

Ružić I. - 1996. Trace metal complexation at heterogeneous binding sites in aquatic systems. Mar. Chem., 53: 1-15.

Ružić I. - 1982. Theoretical aspects of the direct titration of natural waters and its information yield for trace metal speciation. Anal. Chim. Acta, 140: 99-113.

Santana-Casiano J.M., M. Gonzalez-Davila, J. Perez-Peña and F.J. Millero. $-1995 . \mathrm{Pb}^{2+}$ interactions with the marine phytoplankton Dunaliella tertiolecta. Mar. Chem., 48: 115-129.

Scatchard, G. - 1949. The attractions of proteins for small molecules and ions. Ann. N.Y. Acad. Sci., 7: 660-672.

Seritti A., D. Pellegrini, E. Morelli, C. Barghigiani and R. Ferrara. - 1986. Copper complexing capacity of phytoplanktonic cell exudates. Mar. Chem., 18: 351-357.

Sposito G. - 1982. On the use of the Langmuir equation in the interpretation of "Adosrption" phenomena: II. The "Two-Surface" Langmuir equation. Soil. Sci. Soc. Am. J., 46: 1147-1152.

Sunda,W.G. and R.L. Ferguson. - 1983. Sensitivity of natural bacterial communities to additions of copper and to cupric ion activity: a bioassay of copper complexation in sea water. In: C.W. Wong, E. Boyle, K. Bruland, J. Burton and E.D. Goldberg (Eds.) Trace metals in Sea Water. Plenum, New York, NY: 871-891.

Sunda,W.G. and R.R.L. Guillard . - 1976. The relationship between cupric ion activity and the toxicity of copper to phytoplankton. J. Marine Res., 34: 511-529.

van den Berg, C.M.G. - 1982. Determination of copper complexation with natural organic ligands in seawater by equilibration with $\mathrm{MnO}_{2}$. I. Theory. Mar. Chem., 11: 307-322.

van den Berg, C.M.G. - 1984. Determination of copper in sea water by cathodic stripping voltammetry of complexes with cathecol. Anal. Chim. Acta, 16: 195-207.

van den Berg, C.M.G. and J.R. Donat. - 1992. Determination and data evolution of copper complexation in seawater using cathodic stripping voltammetry at varying detection windows. Anal. Chim. Acta, 257: 281-291.

Wu J. and G.W. Luther. - 1995. Complexation of Fe(III) by natural organic ligands in the Northwest Atlantic Ocean by a competitive ligand equilibration method and a kinetic approach. Mar. Chem., 50: 159-177.

Xue, H.B., and L. Sigg. - 1990. Binding of $\mathrm{Cu}(\mathrm{II})$ to algae in a metal buffer. Water Res., 24: 1129-1136.

Xue, H.B., and L. Sigg. - 1993. Free cupric ion concentration and $\mathrm{Cu}(\mathrm{II})$ speciation in a eutrophic lake. Limnol. Oceanogr., 38(6): 1200-1213.

Xue, H.B., and L. Sigg. - 1994. Zinc speciation in lake waters and its determination by ligand exchange with EDTA and differential pulse anodic stripping voltammetry. Anal. Chim. Acta, 284: 505-515.

Xue, H.B., W. Stumm and L. Sigg. - 1988. The binding of heavy metals to algal surfaces. Water Res., 22: 917-926.

Zhou, X. and P.J. Wangersky. - 1985. Copper complexing capacity in cultures of Phaeodactylum tricornutum: diurnal changes. Mar. Chem., 17: 301-312.

Zhou, X. and P.J. Wangersky. - 1989. Production of copper-complexing organic ligands by the marine diatom Phaeodactylum tricornutum in a cage culture turbidostat. Mar. Chem., 26: 239-259. 